\title{
Semi-empirical studies of atomic transition probabilities, oscillator strengths and radiative lifetimes in Hf II
}

\author{
S. Bouazza ${ }^{\text {a,* }}$, P. Quinet $^{\text {b,c }}$, P. Palmeri ${ }^{\text {b }}$ \\ a LISM, E. A. 4695 Université de Reims-Champagne-Ardenne, UFR SEN, BP 1039, F-51687 Reims Cedex 2, France \\ ${ }^{\mathrm{b}}$ Astrophysique et Spectroscopie, Université de Mons, B-7000 Mons, Belgium \\ ' IPNAS, Université de Liège, B-4000 Liège, Belgium
}

\section{A R T I C L E I N F O}

\section{Article history:}

Received 27 February 2015

Accepted 21 April 2015

Available online 30 April 2015

\section{Keywords:}

Atomic structure

Transition probabilities

Oscillator strengths

Lifetimes

Hf II spectrum

\begin{abstract}
A B S T R A C T
Over the past few years, laser induced fluorescence and Fourier Transform techniques have been applied to measure radiative lifetimes and branching fractions in $\mathrm{Hf}$ II in order to derive oscillator strengths and transition probabilities. In the present work, we propose to compare for the first time these experimental data to computed values obtained by two different semi-empirical approaches, respectively based on a parametrization of the oscillator strengths and on a pseudo-relativistic Hartree-Fock model including corepolarization effects. The overall agreement between all sets of data is found to be good. We furthermore give radial integrals of the main atomic transitions in this study: $\langle 5 \mathrm{~d} 6 \mathrm{~s} 6 \mathrm{pl}$ $r^{1}\left|5 d^{2} 6 s\right\rangle=0.1504 \quad(0.0064),\left\langle 6 s^{2} 6 p\left|r^{1}\right| 5 d 6 s^{2}\right\rangle=1.299 \quad(0.012),\left\langle 5 d^{2} 6 p\left|r^{1}\right| 5 d^{2} 6 s\right\rangle=-0.298$ (0.013), $\left\langle 5 \mathrm{~d}^{2} 6 \mathrm{pr}^{1} \mid 5 \mathrm{~d}^{3}\right\rangle=2.025$ (0.027). Finally a new set of oscillator strengths and transition probabilities is reported for many transitions in Hf II.
\end{abstract}

(c) 2015 Elsevier Ltd. All rights reserved.

\section{Introduction}

It is very interesting to study atomic and nuclear properties of refractory elements with $Z=71-78$ and particularly hafnium which has the longest known isotopic chain of 31 isotopes. High-resolution Doppler limited laser spectroscopy has been employed in the past for example to investigate hyperfine structure of selected lines of hafnium in the red spectral region. Using laser induced fluorescence and optogalvanic detection methods, measurements were performed in the plasma of a liquid nitrogen cooled hollow cathode discharge in the atomic spectrum of hafnium [1,2]. Nevertheless our measurements have been limited to low-lying levels due to the high evaporation temperature of this refractory element. Today the development of various methods for producing

\footnotetext{
* Corresponding author.

E-mail address: safa.bouazza@univ-reims.fr (S. Bouazza).
}

intense atomic beams and the introduction of Fourier transform and laser spectroscopic techniques have considerably improved ability for performing any kinds of measurements on refractory elements. Radiative lifetimes, for instance, from laser-induced fluorescence measurements, reaching an accuracy of $5 \%$ were reported for 41 odd-parity levels of Hf II. These lifetimes were combined with branching fractions measured using Fourier transform spectrometry and transition probabilities for 150 lines of Hf II were determined [3]. In another work new and improved radiative lifetimes for eight levels in Hf I and eighteen levels in Hf II, along with oscillator strengths and wavelengths for 195 transitions in Hf II, were presented [4]. Besides these experimental works unfortunately no huge number of theoretical calculations were achieved in the same time like in the case of other ions. For instance the spectrum and extended term analysis of Hf II is limited to two low-lying configurations for odd- and three ones for even-parity levels up to now. The very interesting Kurucz semi-empirical evaluations for oscillator strength, 
lifetimes or atomic transition probabilities are not yet inserted as regards $\mathrm{Hf}$ II in his famous Tables [5]. We propose then to bring our contributions in order to fill up at least partially this lack of theoretical background.

It is worth reminding the astrophysical interest of a reliable knowledge of radiative parameters in lowly ionization stages of hafnium. Indeed, this element has recently been proposed to be used as a stable reference element in nucleocosmochronometry based on the unstable elements thorium and uranium whose radioactive decay timescales are well known [4]. In this latter study, it was also shown that newly Hf abundances in the Sun and 10 metal poor, rprocess-rich halo stars set the stage for improved radioactive stellar age determinations using the Th/Hf chronometer pair. In addition, the observed stellar abundance ratio of $\mathrm{Hf} / \mathrm{Eu}$ was found to be larger than previous estimates of the solar system r-process-only value, suggesting a somewhat larger contribution from the r-process to the production of hafnium.

\section{Oscillator strength and transition probability determination in Hf II}

\subsection{Oscillator strength parametrization method}

In this study we looked first into electric dipole transitions. We had recourse to a semi-empirical method for parametrization of oscillator strengths. The complete details of this method were already described in previous paper [6]; nevertheless let us mention once more that we transformed angular coefficients of the transition matrix from SL coupling to intermediate one, using fine structure eigenvector amplitudes, previously determined [7-9] and presently reanalyzed for odd-parity ones.

For the electric dipole transitions, the weighted oscillator strength $g f$ is related to the line strength $\mathbf{S}[10]$ :

$g f=8 \pi^{2} m c a_{0}^{2} \frac{\sigma}{3 h} \mathbf{S}=303.76 \times 10^{-8} \sigma \mathbf{S}$,

where $a_{0}$ is the Bohr radius, $\sigma=\left|E(\gamma)-E\left(\gamma^{\prime}\right)\right| / h c$ and $h$ is Planck's constant. Let us point out that $E(\gamma)$ is the energy of the initial state. The quantities with primes refer to the final state.

The electric dipole line strength is defined by

$\mathbf{S}=\left|\left\langle\gamma J|| \mathbf{P}^{\mathbf{1}}|| \gamma^{\prime} J^{\prime}\right\rangle\right|^{2}$,

The tensorial operator $\mathbf{P}^{\mathbf{1}}$ in the reduced matrix element represents the electric dipole moment.

For a multiconfiguration system, the wavefunctions $|\gamma J\rangle$ and $\left|\gamma^{\prime} J^{\prime}\right\rangle$ are expanded in terms of a set of basis functions $\mid$ $\psi S L J\rangle$ and $\left|\psi^{\prime} S^{\prime} L^{\prime} J^{\prime}\right\rangle$, respectively:

$|\gamma J\rangle=\sum_{i} c_{i}|\psi S L J\rangle,\left|\gamma^{\prime} J^{\prime}\right\rangle=\sum_{j} c_{j}^{\prime}\left|\psi^{\prime} S^{\prime} L^{\prime} J^{\prime}\right\rangle$

The square root of the line strength may be written in the following form:

$S_{\gamma \gamma^{\prime}}^{1 / 2}=\sum_{i} \sum_{j} c_{i} c_{j}^{\prime}\left\langle\psi S L J\left\|\mathbf{P}^{1}\right\| \psi^{\prime} S^{\prime} L^{\prime} J^{\prime}\right\rangle$
From Eqs. (2) and (5), we can express the $g f$-value as a linear combination:

$$
\begin{aligned}
(g f)^{1 / 2}= & \sum_{n l, n^{\prime} l^{\prime}}\left(303.76 \sigma \times 10^{-8}\right)^{1 / 2} \\
& \times \sum_{i} \sum_{j} c_{i} c_{j}^{\prime}\left\langle\psi S L J\left\|\mathbf{P}^{1}\right\| \psi^{\prime} S^{\prime} L^{\prime} J^{\prime}\right\rangle,
\end{aligned}
$$

where the sum is over all possible transitions ( $n s \leftrightarrow n^{\prime} p$, $\mathrm{nd} \leftrightarrow \mathrm{n}^{\prime} \mathrm{p}$, nd $\leftrightarrow \mathrm{n}^{\prime} \mathrm{f}$ )

The weighted transition probability is [10]

$g A=\left(2 j^{\prime}+1\right) A=64 \pi^{4} e^{2} a \sigma^{3} \mathbf{S} / 3 h=2.0261 \times 10^{-6} \sigma^{3} \mathbf{S}$

where $\sigma$ is given in $\mathrm{cm}^{-1}$ and $\mathbf{S}$ in atomic units of $e^{2} a_{0}^{2}$.

Using Eqs. (1) and (6) one obtains, in $s^{-1}$ :

$g A=0.66702 \sigma^{2} g f$

The extended basis system for odd-parity configurations used in this analysis corresponds to the 6 following configurations: $5 d^{2} 6 p, 5 d 6 s 6 p, 6 s^{2} 6 p, 5 d^{2} 7 p, 5 d 6 s 7 p, 6 s^{2} 7 p$ where configuration mixing is expected to be very strong between the three first cited configurations. Regarding even-parity configurations were involved: $5 d^{1} 6 s^{2}, 5 d^{2} 6 s^{1}, 5 d^{3}, 5 d^{1} 6 s^{1} 7 s^{1}$, $5 d^{2} 7 s^{1}, 5 d^{2} 6 d^{1}$ and $5 d^{2} 8 s^{1}$. As it is commonly the case with the $5 \mathrm{~d}$-elements, Hf II has many low-lying levels below $40,000 \mathrm{~cm}^{-1}$ which are members of the even-parity configurations as well as the odd-parity ones.

Unfortunately due to a small number of experimental energy levels, available in literature only for the two low configurations of this basis system, we fixed the 3 last oddparity configuration fine structure parameter values to their weighted ab-initio data, computed by means of Cowan code [10]. The fitted fine structure parameter values of this extended basis system are shown in Table 1. Transition integrals, treated as free parameters in the least squares fit

Table 1

Fine structure parameter values adopted in the oscillator strength

\begin{tabular}{|c|c|c|c|}
\hline Configuration & $5 d 6 s 6 p$ & $6 s^{2} 6 p$ & $5 d^{2} 6 p$ \\
\hline$E_{\mathrm{av}}$ & $41,980(175)$ & $43,002(527)$ & $50,497(59)$ \\
\hline $\mathrm{F}^{2}(5 \mathrm{~d}, 5 \mathrm{~d})$ & & & $35,093(751)$ \\
\hline$F^{4}(5 d, 5 d)$ & & & $20,016(1013)$ \\
\hline $\mathrm{F}^{2}(5 \mathrm{~d}, 6 \mathrm{p})$ & 16,009 (627) & & $13,995(387)$ \\
\hline$G^{1}(6 s, 6 p)$ & $20,115(920)$ & & \\
\hline$G^{2}(5 d, 6 s)$ & $15,849(980)$ & & \\
\hline$G^{1}(5 d, 6 p)$ & $8811(302)$ & & 8350 (187) \\
\hline$G^{3}(5 d, 6 p)$ & 4502 (928) & & 3398 (541) \\
\hline$\zeta_{5 d}$ & $1668(68)$ & & $1424(39)$ \\
\hline$\zeta_{6 p}$ & $4180(130)$ & 4476 (310) & 3426 (102) \\
\hline$\alpha$ & $-14(7)$ & & $-14(7)$ \\
\hline$\beta$ & $-41(38)$ & & $-41(38)$ \\
\hline \multicolumn{4}{|c|}{ Configuration interaction parameters } \\
\hline & $6 s^{2} 6 p-5 d^{2} 6 p$ & $5 d 6 s 6 p-6 s^{2} 6 p$ & $5 d 6 s 6 p-5 d^{2} 6 p$ \\
\hline$D^{2}(6 s 6 s, 5 d 5 d)$ & $18,395(602)$ & & \\
\hline$D^{2}(5 d 6 p, 6 s 6 p)$ & & $-15,745$ (707) & \\
\hline $\mathrm{E}^{1}(5 \mathrm{~d} 6 \mathrm{p}, 6 \mathrm{~s} 6 \mathrm{p})$ & & $-14,441(477)$ & \\
\hline $\mathrm{D}^{2}(6 \mathrm{~s} 6 \mathrm{p}, 5 \mathrm{~d} 6 \mathrm{p})$ & & & $-12,920(321)$ \\
\hline$E^{1}(6 s 6 p, 6 p 5 d)$ & & & $-11,878(238)$ \\
\hline$D^{2}(5 d 6 s, 5 d 5 d)$ & & & $-20,488(397)$ \\
\hline
\end{tabular}
parametrization method. All values are given in $\mathrm{cm}^{-1}$. 
to experimental oscillator strength (gf) values, were then extracted; we give the main deduced values in Table 2. The values of the six remaining integrals although predicted by

Table 2

Transition radial integrals obtained in the fitting procedure used in the oscillator strength parametrization method.

\begin{tabular}{lcl}
\hline Transition & Value & Uncertainty \\
\hline$\left\langle 5 \mathrm{~d} 6 \mathrm{~s} 6 \mathrm{p}\left|\mathrm{r}^{1}\right| 5 \mathrm{~d} 6 \mathrm{~s}^{2}\right\rangle$ & -0.267 & 0.011 \\
$\left\langle 5 \mathrm{~d} 6 \mathrm{~s} 6 \mathrm{p}\left|\mathrm{r}^{1}\right| 5 \mathrm{~d}^{2} 6 \mathrm{~s}\right\rangle$ & 0.1504 & 0.0064 \\
$\left\langle 5 \mathrm{~d} 6 \mathrm{~s} 6 \mathrm{p}\left|\mathrm{r}^{1}\right| 5 \mathrm{~d} 6 \mathrm{~s} 7 \mathrm{~s}\right\rangle$ & 0.123 & 0.009 \\
$\left\langle 6 \mathrm{~s}^{2} 6 \mathrm{p}\left|\mathrm{r}^{1}\right| 5 \mathrm{~d} 6 \mathrm{~s}^{2}\right\rangle$ & 1.299 & 0.012 \\
$\left\langle 5 \mathrm{~d}^{2} 6 \mathrm{p}\left|\mathrm{r}^{1}\right| 5 \mathrm{~d}^{2} 6 \mathrm{~s}\right\rangle$ & -0.298 & 0.013 \\
$\left\langle 5 \mathrm{~d}^{2} 6 \mathrm{p}\left|\mathrm{r}^{1}\right| 5 \mathrm{~d}^{3}\right\rangle$ & 2.025 & 0.027 \\
$\left\langle 5 \mathrm{~d}^{2} 6 \mathrm{p}\left|\mathrm{r}^{1}\right| 5 \mathrm{~d}^{2} 7 \mathrm{~s}\right\rangle$ & -0.135 & 0.009 \\
$\left\langle 5 \mathrm{~d}^{2} 6 \mathrm{p}\left|\mathrm{r}^{1}\right| 5 \mathrm{~d}^{2} 6 \mathrm{~d}\right\rangle$ & 0.641 & 0.026 \\
$\left\langle 5 \mathrm{~d}^{2} 6 \mathrm{p}\left|\mathrm{r}^{1}\right| 5 \mathrm{~d}^{2} 8 \mathrm{~s}\right\rangle$ & -0.038 & 0.015 \\
$\left\langle 5 \mathrm{~d} 6 \mathrm{~s} 7 \mathrm{p}\left|\mathrm{r}^{1}\right| 5 \mathrm{~d} 6 \mathrm{~s}^{2}\right\rangle$ & -0.069 & 0.030 \\
$\left\langle 5 \mathrm{~d} 6 \mathrm{~s} 7 \mathrm{p}\left|\mathrm{r}^{1}\right| 5 \mathrm{~d}^{2} 6 \mathrm{~s}\right\rangle$ & -0.023 & 0.015 \\
$\left\langle 5 \mathrm{~d} 6 \mathrm{~s} 7 \mathrm{p}\left|\mathrm{r}^{1}\right| 5 \mathrm{~d} 6 \mathrm{~s} 7 \mathrm{~s}\right\rangle$ & -0.37 & 0.03 \\
\hline
\end{tabular}

Table 3

Optimized radial parameter values (in $\mathrm{cm}^{-1}$ ) adopted in the $\mathrm{HFR}+\mathrm{CPOL}$ method.

\begin{tabular}{|c|c|c|c|c|}
\hline Configuration & Parameter & $\begin{array}{l}\text { Ab initio } \\
\text { value }\end{array}$ & $\begin{array}{l}\text { Fitted } \\
\text { value }\end{array}$ & Ratio \\
\hline \multicolumn{5}{|l|}{ Even parity } \\
\hline \multirow[t]{2}{*}{$5 \mathrm{~d} 6 \mathrm{~s}^{2}$} & $E_{\mathrm{av}}$ & 9213 & 9842 & \\
\hline & $\zeta_{5 d}$ & 1833 & 1528 & 0.834 \\
\hline \multirow[t]{4}{*}{$5 d^{3}$} & $E_{\mathrm{av}}$ & 30,110 & 30,588 & \\
\hline & $\mathrm{F}^{2}(5 \mathrm{~d}, 5 \mathrm{~d})$ & 43,338 & 33,392 & 0.769 \\
\hline & $\mathrm{F}^{4}(5 \mathrm{~d}, 5 \mathrm{~d})$ & 27,936 & 19,782 & 0.708 \\
\hline & $\zeta_{5 d}$ & 1468 & 1207 & 0.822 \\
\hline \multirow[t]{5}{*}{$5 d^{2} 6 s$} & Eav & 14,747 & 14,921 & \\
\hline & $\mathrm{F}^{2}(5 \mathrm{~d}, 5 \mathrm{~d})$ & 46,353 & 36,336 & 0.784 \\
\hline & $\mathrm{F}^{4}(5 \mathrm{~d}, 5 \mathrm{~d})$ & 30,087 & 21,182 & 0.704 \\
\hline & $\zeta_{5 d}$ & 1645 & 1363 & 0.828 \\
\hline & $\mathrm{G}^{2}(5 \mathrm{~d}, 6 \mathrm{~s})$ & 20,915 & 18,287 & 0.874 \\
\hline \multicolumn{5}{|l|}{ Odd parity } \\
\hline \multirow[t]{8}{*}{$5 d^{2} 6 p$} & $E_{\mathrm{av}}$ & 48,896 & 51,067 & \\
\hline & $\mathrm{F}^{2}(5 \mathrm{~d}, 5 \mathrm{~d})$ & 47,606 & 37,187 & 0.781 \\
\hline & $\mathrm{F}^{4}(5 \mathrm{~d}, 5 \mathrm{~d})$ & 30,993 & 21,859 & 0.705 \\
\hline & $\zeta_{5 d}$ & 1715 & 1508 & 0.879 \\
\hline & $\zeta_{6 p}$ & 2611 & 3224 & 1.235 \\
\hline & $\mathrm{F}^{2}(5 \mathrm{~d}, 6 \mathrm{p})$ & 19,038 & 14,554 & 0.764 \\
\hline & $\mathrm{G}^{1}(5 \mathrm{~d}, 6 \mathrm{p})$ & 11,327 & 8943 & 0.790 \\
\hline & $G^{3}(5 d, 6 p)$ & 8343 & 5048 & 0.605 \\
\hline \multirow[t]{8}{*}{$5 d 6 s 6 p$} & $E_{\mathrm{av}}$ & 40,857 & 43,030 & \\
\hline & $\zeta_{5 \mathrm{~d}}$ & 1895 & 1626 & 0.858 \\
\hline & $\zeta_{6 p}$ & 3172 & 4218 & 1.330 \\
\hline & $\mathrm{F}^{2}(5 \mathrm{~d}, 6 \mathrm{p})$ & 20,338 & 16,186 & 0.796 \\
\hline & $G^{2}(5 d, 6 s)$ & 20,628 & 17,568 & 0.852 \\
\hline & $\mathrm{G}^{1}(5 \mathrm{~d}, 6 \mathrm{p})$ & 11,351 & 10,056 & 0.886 \\
\hline & $G^{3}(5 d, 6 p)$ & 8600 & 5340 & 0.621 \\
\hline & $\mathrm{G}^{1}(6 \mathrm{~s}, 6 \mathrm{p})$ & 33,422 & 23,379 & 0.700 \\
\hline \multirow[t]{2}{*}{$6 s^{2} 6 p$} & $E_{\mathrm{av}}$ & 44,825 & 46,283 & \\
\hline & $\zeta_{6 p}$ & 3812 & 4756 & 1.2476 \\
\hline \multirow[t]{3}{*}{$5 d^{2} 6 p-5 d 6 s 6 p$} & $D^{2}(5 d 5 d, 5 d 6 s)$ & $-25,393$ & $-20,032$ & 0.789 \\
\hline & $D^{2}(5 d 6 p, 6 s 6 p)$ & $-19,110$ & $-15,075$ & 0.789 \\
\hline & $\mathrm{E}^{1}(5 \mathrm{~d} 6 \mathrm{p}, 6 \mathrm{~s} 6 \mathrm{p})$ & $-18,845$ & $-14,866$ & 0.789 \\
\hline $5 d^{2} 6 p-6 s^{2} 6 p$ & $D^{2}(5 d 5 d, 6 s 6 s)$ & 23,298 & 25,865 & 1.110 \\
\hline \multirow[t]{2}{*}{$5 d 6 s 6 p-6 s^{2} 6 p$} & $\mathrm{D}^{2}(5 \mathrm{~d} 6 \mathrm{p}, 6 \mathrm{~s} 6 \mathrm{p})$ & $-19,733$ & $-13,863$ & 0.703 \\
\hline & $E^{1}(5 d 6 p, 6 s 6 p)$ & $-19,274$ & $-13,541$ & 0.703 \\
\hline
\end{tabular}

theory but expected to be small in this study were fixed to zero and then are not listed in this Table 2.

\subsection{Pseudo-relativistic Hartree-Fock method with core- polarization corrections}

In the present work, we also used the pseudo-relativistic Hartree-Fock (HFR) approach of Cowan [10] in which we have incorporated core-polarization effects by means of a model potential and a correction to the dipole operator (HFR+CPOL: see e.g. [11,12]). In a previous work on the isoelectronic ion Ta III [13], an excellent agreement was obtained between HFR + CPOL lifetimes and the accurate values measured by time-resolved laser-induced-fluorescence spectroscopy. We have adopted the same physical model here considering a set of 43 configurations: $5 d 6 s^{2}+5 d^{3}+5 d^{2} 6 s+5 d^{2} 6 d+5 d 6 p^{2}+5 d 6 d^{2}+5 d 5 f^{2}+5 d 6 f^{2}-$ $+5 d 6 s 6 d+5 d 6 p 5 f+5 d 6 p 6 f+5 d 5 f 6 f+6 s^{2} 6 d+6 s 6 p^{2}+6 p^{2} 6-$ $d+6 s 6 d^{2}+6 d^{3}+6 s 5 f^{2}+6 d 5 f^{2}+6 s 6 f^{2}+6 d 6 f^{2}$ (even parity)

Table 4

Comparison between available experimental radiative lifetimes and the results obtained in the present work using the HFR +CPOL model. All values are given in ns.

\begin{tabular}{|c|c|c|c|c|}
\hline$E\left(\mathrm{~cm}^{-1}\right)$ & $J$ & Lundqvist et al. [3] & Lawler et al. [4] & $\mathrm{HFR}+\mathrm{CPOL}$ \\
\hline $28,068.79$ & 1.5 & & $39.4 \pm 2.0$ & 37.5 \\
\hline $29,160.04$ & 0.5 & & $43.8 \pm 2.2$ & 44.6 \\
\hline $29,405.12$ & 2.5 & $29.7 \pm 2.4$ & $26.4 \pm 1.3$ & 24.4 \\
\hline $31,784.16$ & 1.5 & $21.8 \pm 1.8$ & $18.9 \pm 0.9$ & 19.3 \\
\hline $33,136.20$ & 0.5 & & $17.6 \pm 0.9$ & 18.0 \\
\hline $33,180.92$ & 2.5 & $18.1 \pm 1.5$ & $16.3 \pm 0.8$ & 15.4 \\
\hline $33,776.24$ & 3.5 & $34.8 \pm 2.5$ & $32.4 \pm 1.6$ & 32.4 \\
\hline $34,123.93$ & 1.5 & $24.5 \pm 2.5$ & $23.3 \pm 1.2$ & 17.5 \\
\hline $34,355.13$ & 2.5 & $16.2 \pm 1.4$ & $14.7 \pm 0.7$ & 13.0 \\
\hline $34,942.36$ & 2.5 & $8.4 \pm 0.5$ & $8.0 \pm 0.4$ & 7.1 \\
\hline $36,373.42$ & 1.5 & & $17.7 \pm 0.9$ & 19.0 \\
\hline $36,882.49$ & 3.5 & & $16.7 \pm 0.8$ & 18.8 \\
\hline $37,885.90$ & 1.5 & & $3.4 \pm 0.2$ & 3.5 \\
\hline $38,185.67$ & 4.5 & & $47.2 \pm 2.4$ & 56.5 \\
\hline $38,398.56$ & 0.5 & & $28.3 \pm 1.4$ & 30.9 \\
\hline $38,498.53$ & 3.5 & $5.1 \pm 0.3$ & $5.0 \pm 0.3$ & 4.1 \\
\hline $38,578.63$ & 2.5 & & $5.2 \pm 0.3$ & 4.5 \\
\hline $39,226.46$ & 1.5 & & $29.1 \pm 1.5$ & 28.3 \\
\hline $40,506.86$ & 2.5 & & $12.4 \pm 0.6$ & 10.6 \\
\hline $41,406.86$ & 3.5 & & $5.2 \pm 0.3$ & 4.1 \\
\hline $41,761.24$ & 2.5 & & $2.9 \pm 0.2$ & 2.7 \\
\hline $42,391.09$ & 4.5 & & $4.2 \pm 0.2$ & 3.4 \\
\hline $42,518.10$ & 1.5 & $2.3 \pm 0.2$ & $2.15 \pm 0.20$ & 1.9 \\
\hline $42,770.56$ & 1.5 & $2.6 \pm 0.2$ & $2.6 \pm 0.2$ & 2.3 \\
\hline $43,044.26$ & 0.5 & $1.8 \pm 0.2$ & $1.75 \pm 0.20$ & 1.4 \\
\hline $43,680.75$ & 2.5 & $3.3 \pm 0.2$ & $3.2 \pm 0.2$ & 2.0 \\
\hline $43,900.56$ & 2.5 & & $2.7 \pm 0.2$ & 4.1 \\
\hline $44,399.96$ & 3.5 & & $4.1 \pm 0.2$ & 2.3 \\
\hline $44,690.72$ & 3.5 & & $2.7 \pm 0.2$ & 4.4 \\
\hline $45,643.25$ & 0.5 & $2.2 \pm 0.2$ & $2.40 \pm 0.2$ & 2.1 \\
\hline $46,124.89$ & 4.5 & & $2.8 \pm 0.2$ & 2.4 \\
\hline $46,209.05$ & 5.5 & & $3.3 \pm 0.2$ & 2.8 \\
\hline $46,495.37$ & 0.5 & $2.7 \pm 0.2$ & $2.7 \pm 0.2$ & 2.4 \\
\hline $46,674.36$ & 1.5 & $2.1 \pm 0.2$ & $2.00 \pm 0.20$ & 1.8 \\
\hline $47,157.57$ & 3.5 & & $3.2 \pm 0.2$ & 2.7 \\
\hline $47,904.39$ & 2.5 & $2.0 \pm 0.2$ & $2.05 \pm 0.20$ & 1.9 \\
\hline $47,973.56$ & 1.5 & & $2.50 \pm 0.20$ & 2.1 \\
\hline $48,930.75$ & 3.5 & & $2.10 \pm 0.20$ & 1.7 \\
\hline $49,005.64$ & 2.5 & & $2.6 \pm 0.20$ & 2.0 \\
\hline $49,840.47$ & 4.5 & $3.1 \pm 0.2$ & $3.1 \pm 0.2$ & 2.6 \\
\hline $52,340.08$ & 3.5 & $1.9 \pm 0.2$ & & 1.6 \\
\hline $53,227.27$ & 3.5 & & $2.55 \pm 0.20$ & 2.1 \\
\hline
\end{tabular}




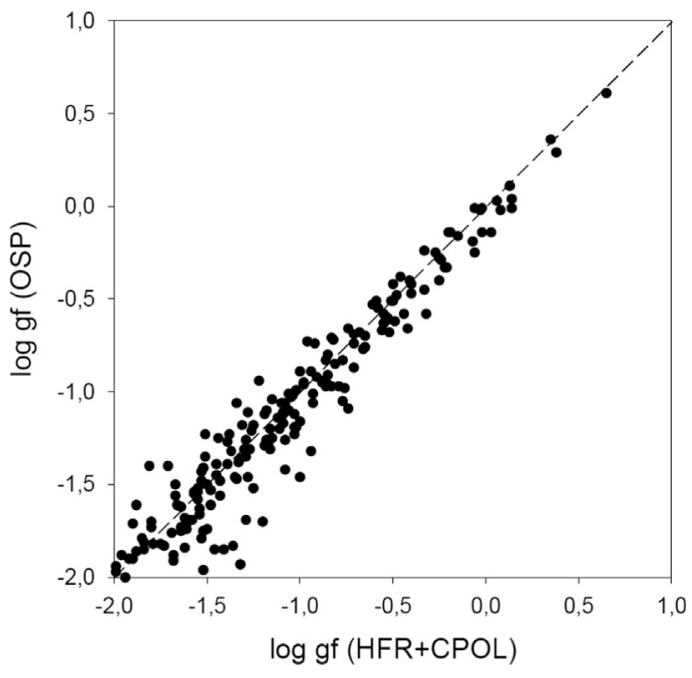

Fig. 1. Comparison between the log gf-values obtained in the present work with the oscillator strength parametrization method (OSP) and the pseudo-relativistic Hartree-Fock approach including core-polarization effects $(\mathrm{HFR}+\mathrm{CPOL})$. Only transitions with $\log g f>-2$ are shown in the figure.

and $5 d^{2} 6 p+5 d^{2} 5 f+5 d^{2} 6 f+5 d 6 s 6 p+5 d 6 s 5 f+5 d 6 s 6 f+5 d 6 p$ $6 d+5 d 6 d 5 f+5 d 6 d 6 f+6 s^{2} 6 p+6 s^{2} 5 f+6 s^{2} 6 f+6 p^{2} 5 f+6 p^{2} 6 f-$ $+6 p^{3}+6 p 6 d^{2}+6 d^{2} 5 f+6 d^{2} 6 f+6 p 5 f^{2}+6 p 6 f^{2}+5 f^{2} 6 f+5 f 6 f^{2}$ (odd parity). For the core-polarization corrections, we considered an erbium - like core surrounded by 3 valence electrons. The adopted dipole polarizabilty was $\alpha_{d}=3.85 \mathrm{a}_{0}^{3}$ which corresponds to a Hf V ionic core [14]. The cut-off radius used was the HFR mean radius of the outermost core orbital 5p, i.e. $r_{c}=1.33 \mathrm{a}_{0}$. To optimize the calculation of oscillator strengths, the HFR +CPOL method was combined with a semi-empirical fitting of the radial parameters minimizing the discrepancies between the computed energies and the experimental values compiled at the National Institute of Standards and Technology (NIST) [15] for the $5 \mathrm{~d}_{6} \mathrm{~s}^{2}$, $5 d^{3}$ and $5 d^{2} 6$ s even-parity configurations and published by Wyart and Blaise [8] for the $5 d^{2} 6 p, 5 d 6 s 6 p$ and $6 s^{2} 6 p$ oddparity configurations. The adopted values for the radial parameters are reported in Table 3. At the end of the fitting process, the average deviations between experimental and computed energy levels were found to be equal to $118 \mathrm{~cm}^{-1}$ (even parity) and $140 \mathrm{~cm}^{-1}$ (odd parity). These calculations can be seen as an extension of our previous similar works on singly ionized 5d-transition atoms Ta II [16], W II [17], Re II [18], Os II [19], Ir II [20], Pt II [21], and Au II [22].

\section{Results and discussion}

In Table 4, we give the oscillator strengths computed in the present work using both methods described here above for a sample of about 300 selected Hf II transitions. More precisely, only lines with HFR+CPOL log gf-values greater than -1.0 are listed in this table in which are also reported the available experimental data $[3,4]$. When comparing the results obtained with our two computational approaches, one can notice an overall good agreement (within 20-30\%). This general agreement is illustrated in Fig. 1 where oscillator strengths are compared for all transitions with $\log g f>-2.0$. It is not worth making the comparison for weaker transitions since most of them were found to be affected by large cancellation effects in the HFR + CPOL line strength calculations, indicating that those results could be affected by large uncertainties.

When looking at Table 4, we also note that the agreement between the oscillator strengths computed in the present work and the available experimental data is satisfactory, all sets of data standing within $30 \%$ for most transitions. Much larger differences are nevertheless observed for some lines, in particular for those situated at $2288.632,2677.555$ and $4245.845 \AA$ for which the discrepancies between the HFR + CPOL $g f$-values and the experimental data [3] can reach several orders of magnitude. However, it is interesting to note that, for the same transitions, the results obtained in our work using the oscillator strength parametrization method show similar discrepancies with experiment.

Finally, in Table 5, we report on the comparison between our HFR + CPOL radiative lifetimes and the accurate experimental values recently measured using laserinduced fluorescence spectroscopy by Lundqvist et al. [3] and Lawler et al. [4]. As seen from this table, a very good agreement is also observed, the ratios $\tau_{\mathrm{HFR}+\mathrm{CPOL}} / \tau_{\mathrm{EXP}}$ being found to be equal to $0.84 \pm 0.08$ and $0.93 \pm 0.19$ when considering the experimental lifetimes from [3] and [4], respectively.

\section{Conclusion}

Very interesting experimental data of Hf II lifetimes, transition probabilities and oscillator strengths were given some years ago but unfortunately without any theoretical background. In the present work we have compared these experimental values with computed ones obtained using two independent semi-empirical methods. We have confirmed the well-founded basis of these experimental sets of data. In many analyses of physical and astrophysical problems linked to fundamental characteristics of atomic and ionic excited states, a large number of radial transition integrals are required. Such parameters are given in this paper for the twelve main Hf II transition arrays. Despite the scarcity of Hf II energy levels, their eigenvector amplitudes could nevertheless be used to determine the angular part of the matrix element: $\left\langle\psi S L J\left\|\mathbf{P}^{\mathbf{1}}\right\| \psi^{\prime} S^{\prime} L^{\prime} J^{\prime}\right\rangle$ (see Eq. (5)), recurring to Racah Algebra as in the cases of hyperfine structure and isotope shift studies. As for the latter fields we have to confess that we prefer to have at our disposal more experimental energy levels and then more accurate eigenvectors and less oscillator strength values than the opposite situation as in the case of Hf II. We have to point out, according to our knowledge that all these transition radial integral values are given for the first time. Finally our investigation of singly ionized hafnium was extended by comparing our calculated oscillator strengths with available experimental data and by predicting new values for a rather large number of transitions. We hope that this work, in the image of our previous studies [16-24], will constitute a good stimulus and a 
Table 5

Oscillator strengths for selected transitions $(\log g f(\mathrm{HFR}+\mathrm{CPOL})>-1.0)$ in Hf II.

\begin{tabular}{|c|c|c|c|c|c|c|c|c|c|c|}
\hline \multirow[t]{2}{*}{$\lambda(\AA)^{\mathrm{a}}$} & \multicolumn{3}{|c|}{ Lower level $^{\mathrm{b}}$} & \multicolumn{3}{|c|}{ Upper level $^{\mathrm{b}}$} & \multicolumn{2}{|c|}{$\begin{array}{c}\log g f \\
\text { Experiment }\end{array}$} & \multicolumn{2}{|c|}{$\begin{array}{l}\log g f^{c} \\
\text { This work }\end{array}$} \\
\hline & $E\left(\mathrm{~cm}^{-1}\right)$ & & $J$ & $E\left(\mathrm{~cm}^{-1}\right)$ & & $J$ & [3] & [4] & OSP & $\mathrm{HFR}+\mathrm{CPOL}$ \\
\hline 1774.757 & 0 & (e) & 1.5 & 56,346 & (o) & 1.5 & & & & -0.23 \\
\hline 1815.640 & 0 & (e) & 1.5 & 55,077 & (o) & 1.5 & & & & -0.97 \\
\hline 1815.985 & 3051 & (e) & 2.5 & 58,117 & (o) & 2.5 & & & & -0.96 \\
\hline 1869.365 & 0 & (e) & 1.5 & 53,494 & (o) & 1.5 & & & & -0.84 \\
\hline 1919.518 & 0 & (e) & 1.5 & 52,096 & (o) & 0.5 & & & & -0.61 \\
\hline 1922.111 & 3051 & (e) & 2.5 & 55,077 & (o) & 1.5 & & & & -0.71 \\
\hline 1922.727 & 3051 & (e) & 2.5 & 55,060 & (o) & 2.5 & & & & -0.23 \\
\hline 1955.662 & 0 & (e) & 1.5 & 51,134 & (o) & 1.5 & & & & -0.90 \\
\hline 1963.804 & 3051 & (e) & 2.5 & 53,972 & (o) & 3.5 & & & & -0.79 \\
\hline 1964.242 & 0 & (e) & 1.5 & 50,910 & (o) & 2.5 & & & & -0.05 \\
\hline 1993.799 & 4905 & (e) & 2.5 & 55,060 & (o) & 2.5 & & & & -0.85 \\
\hline 2012.776 & 3051 & (e) & 2.5 & 52,717 & (o) & 1.5 & & & & -0.52 \\
\hline 2028.195 & 3051 & (e) & 2.5 & 52,340 & (o) & 3.5 & -0.334 & & -0.14 & -0.19 \\
\hline 2088.791 & 3051 & (e) & 2.5 & 50,910 & (o) & 2.5 & & & & -0.74 \\
\hline 2107.476 & 4905 & (e) & 2.5 & 52,340 & (o) & 3.5 & -0.915 & & -0.69 & -0.71 \\
\hline 2156.435 & 6344 & (e) & 3.5 & 52,703 & (o) & 2.5 & & & & -0.97 \\
\hline 2178.923 & 3051 & (e) & 2.5 & 48,931 & (o) & 3.5 & & & & -0.38 \\
\hline 2212.463 & 8362 & (e) & 4.5 & 53,546 & (o) & 4.5 & & & & -0.72 \\
\hline 2254.000 & 3645 & (e) & 1.5 & 47,996 & (o) & 0.5 & & & & -0.32 \\
\hline 2255.166 & 3645 & (e) & 1.5 & 47,974 & (o) & 1.5 & & & & -0.71 \\
\hline 2257.897 & 12,070 & (e) & 2.5 & 56,346 & (o) & 1.5 & & & & -0.67 \\
\hline 2266.529 & 3051 & (e) & 2.5 & 47,158 & (o) & 3.5 & & & & -0.99 \\
\hline 2266.832 & 4905 & (e) & 2.5 & 49,006 & (o) & 2.5 & & & & -0.31 \\
\hline 2273.153 & 8362 & (e) & 4.5 & 52,340 & (o) & 3.5 & -0.628 & & -0.58 & -0.32 \\
\hline 2277.172 & 0 & (e) & 1.5 & 43,901 & (o) & 2.5 & & & & -0.73 \\
\hline 2284.592 & 14,359 & (e) & 1.5 & 58,117 & (o) & 2.5 & & & & -0.54 \\
\hline 2288.632 & 0 & (e) & 1.5 & 43,681 & (o) & 2.5 & -2.544 & & -0.62 & -0.49 \\
\hline 2321.158 & 4905 & (e) & 2.5 & 47,974 & (o) & 1.5 & & & & -0.32 \\
\hline 2322.477 & 0 & (e) & 1.5 & 43,044 & (o) & 0.5 & -0.140 & & -0.14 & -0.02 \\
\hline 2323.262 & 3645 & (e) & 1.5 & 46,674 & (o) & 1.5 & -0.550 & & -0.58 & -0.44 \\
\hline 2324.514 & 12,070 & (e) & 2.5 & 55,077 & (o) & 1.5 & & & & -0.42 \\
\hline 2324.892 & 4905 & (e) & 2.5 & 47,904 & (o) & 2.5 & -0.315 & & -0.45 & -0.33 \\
\hline 2332.967 & 3645 & (e) & 1.5 & 46,495 & (o) & 0.5 & -0.946 & & -0.85 & -0.81 \\
\hline 2343.327 & 6344 & (e) & 3.5 & 49,006 & (o) & 2.5 & & & & -0.07 \\
\hline 2347.448 & 6344 & (e) & 3.5 & 48,931 & (o) & 3.5 & & & & -0.07 \\
\hline 2351.220 & 0 & (e) & 1.5 & 42,518 & (o) & 1.5 & -0.331 & & -0.16 & -0.15 \\
\hline 2371.414 & 12,921 & (e) & 1.5 & 55,077 & (o) & 1.5 & & & & -0.79 \\
\hline 2372.351 & 12,921 & (e) & 1.5 & 55,060 & (o) & 2.5 & & & & -0.95 \\
\hline 2380.306 & 3645 & (e) & 1.5 & 45,643 & (o) & 0.5 & -0.513 & & -0.66 & -0.42 \\
\hline 2381.002 & 14,359 & (e) & 1.5 & 56,346 & (o) & 1.5 & & & & -0.33 \\
\hline 2393.185 & 12,921 & (e) & 1.5 & 54,694 & (o) & 2.5 & & & & -0.05 \\
\hline 2393.362 & 4905 & (e) & 2.5 & 46,674 & (o) & 1.5 & -0.136 & & -0.25 & -0.06 \\
\hline 2393.836 & 0 & (e) & 1.5 & 41,761 & (o) & 2.5 & & & & -0.30 \\
\hline 2400.815 & 3051 & (e) & 2.5 & 44,691 & (o) & 3.5 & & & & -0.55 \\
\hline 2403.609 & 13,486 & (e) & 2.5 & 55,077 & (o) & 1.5 & & & & -0.84 \\
\hline 2404.572 & 13,486 & (e) & 2.5 & 55,060 & (o) & 2.5 & & & & -0.27 \\
\hline
\end{tabular}




\begin{tabular}{|c|c|c|c|c|c|c|c|c|c|c|}
\hline \multirow[t]{2}{*}{$\lambda(\AA)^{a}$} & \multicolumn{3}{|c|}{ Lower level $^{\mathrm{b}}$} & \multicolumn{3}{|c|}{ Upper level $^{\mathrm{b}}$} & \multicolumn{2}{|c|}{$\begin{array}{c}\log g f \\
\text { Experiment }\end{array}$} & \multicolumn{2}{|c|}{$\begin{array}{l}\log g f^{c} \\
\text { This work }\end{array}$} \\
\hline & $E\left(\mathrm{~cm}^{-1}\right)$ & & $J$ & $E\left(\mathrm{~cm}^{-1}\right)$ & & $J$ & [3] & [4] & OSP & $\mathrm{HFR}+\mathrm{CPOL}$ \\
\hline 2405.425 & 6344 & (e) & 3.5 & 47,904 & (o) & 2.5 & 0.052 & & -0.14 & 0.03 \\
\hline 2406.447 & 11,952 & (e) & 0.5 & 53,494 & (o) & 1.5 & & & & -0.18 \\
\hline 2410.142 & 8362 & (e) & 4.5 & 49,840 & (o) & 4.5 & 0.024 & & 0.04 & 0.14 \\
\hline 2413.347 & 12,070 & (e) & 2.5 & 53,494 & (o) & 1.5 & & & & -0.63 \\
\hline 2415.962 & 11,952 & (e) & 0.5 & 53,331 & (o) & 0.5 & & & & -0.85 \\
\hline 2417.699 & 3051 & (e) & 2.5 & 44,400 & (o) & 3.5 & & & & -0.97 \\
\hline 2425.978 & 13,486 & (e) & 2.5 & 54,694 & (o) & 2.5 & & & & -0.22 \\
\hline 2428.994 & 12,070 & (e) & 2.5 & 53,227 & (o) & 3.5 & & & & -0.34 \\
\hline 2433.575 & 17,369 & (e) & 2.5 & 58,448 & (o) & 3.5 & & & & 0.25 \\
\hline 2434.772 & 17,389 & (e) & 4.5 & 58,448 & (o) & 3.5 & & & & -0.63 \\
\hline 2449.444 & 6344 & (e) & 3.5 & 47,158 & (o) & 3.5 & & & & -0.58 \\
\hline 2452.296 & 11,952 & (e) & 0.5 & 52,717 & (o) & 1.5 & & & & -0.81 \\
\hline 2453.333 & 17,369 & (e) & 2.5 & 58,117 & (o) & 2.5 & & & & 0.15 \\
\hline 2453.998 & 17,711 & (e) & 3.5 & 58,448 & (o) & 3.5 & & & & -0.56 \\
\hline 2455.198 & 14,359 & (e) & 1.5 & 55,077 & (o) & 1.5 & & & & -0.64 \\
\hline 2460.499 & 3051 & (e) & 2.5 & 43,681 & (o) & 2.5 & -0.254 & & 0.03 & 0.06 \\
\hline 2463.938 & 12,921 & (e) & 1.5 & 53,494 & (o) & 1.5 & & & & -0.50 \\
\hline 2464.192 & 8362 & (e) & 4.5 & 48,931 & (o) & 3.5 & & & & 0.32 \\
\hline 2465.070 & 15,084 & (e) & 3.5 & 55,639 & (o) & 4.5 & & & & -0.26 \\
\hline 2469.188 & 13,486 & (e) & 2.5 & 53,972 & (o) & 3.5 & & & & 0.13 \\
\hline 2473.914 & 12,921 & (e) & 1.5 & 53,331 & (o) & 0.5 & & & & -0.30 \\
\hline 2478.542 & 14,359 & (e) & 1.5 & 54,694 & (o) & 2.5 & & & & -0.91 \\
\hline 2481.437 & 17,830 & (e) & 1.5 & 58,117 & (o) & 2.5 & & & & -0.55 \\
\hline 2483.357 & 3645 & (e) & 1.5 & 43,901 & (o) & 2.5 & & & & -0.97 \\
\hline 2496.993 & 3645 & (e) & 1.5 & 43,681 & (o) & 2.5 & -0.581 & & -0.55 & -0.58 \\
\hline 2500.741 & 15,084 & (e) & 3.5 & 55,060 & (o) & 2.5 & & & & -0.30 \\
\hline 2512.699 & 4905 & (e) & 2.5 & 44,691 & (o) & 3.5 & & & & -0.64 \\
\hline 2512.965 & 12,921 & (e) & 1.5 & 52,703 & (o) & 2.5 & & & & 0.04 \\
\hline 2513.035 & 6344 & (e) & 3.5 & 46,125 & (o) & 4.5 & & & & 0.11 \\
\hline 2515.491 & 13,486 & (e) & 2.5 & 53,227 & (o) & 3.5 & & & & 0.01 \\
\hline 2516.886 & 3051 & (e) & 2.5 & 42,771 & (o) & 1.5 & 0.039 & & -0.01 & 0.14 \\
\hline 2521.484 & 17,830 & (e) & 1.5 & 57,478 & (o) & 0.5 & & & & -0.22 \\
\hline 2531.198 & 4905 & (e) & 2.5 & 44,400 & (o) & 3.5 & & & & 0.02 \\
\hline 2548.181 & 13,486 & (e) & 2.5 & 52,717 & (o) & 1.5 & & & & -0.34 \\
\hline 2551.391 & 17,389 & (e) & 4.5 & 56,572 & (o) & 5.5 & & & & 0.48 \\
\hline 2551.434 & 11,952 & (e) & 0.5 & 51,134 & (o) & 1.5 & & & & -0.25 \\
\hline 2551.853 & 12,921 & (e) & 1.5 & 52,096 & (o) & 0.5 & & & & -0.97 \\
\hline 2559.191 & 12,070 & (e) & 2.5 & 51,134 & (o) & 1.5 & & & & -0.40 \\
\hline 2570.704 & 15,084 & (e) & 3.5 & 53,972 & (o) & 3.5 & & & & -0.60 \\
\hline 2571.679 & 3645 & (e) & 1.5 & 42,518 & (o) & 1.5 & -0.071 & & -0.19 & -0.07 \\
\hline 2572.942 & 13,486 & (e) & 2.5 & 52,340 & (o) & 3.5 & -1.154 & & -3.03 & -0.85 \\
\hline 2573.910 & 12,070 & (e) & 2.5 & 50,910 & (o) & 2.5 & & & & 0.19 \\
\hline 2576.826 & 8362 & (e) & 4.5 & 47,158 & (o) & 3.5 & & & & -0.13 \\
\hline 2578.149 & 4905 & (e) & 2.5 & 43,681 & (o) & 2.5 & -0.617 & & -0.33 & -0.22 \\
\hline 2582.515 & 3051 & (e) & 2.5 & 41,761 & (o) & 2.5 & & & & -0.71 \\
\hline 2595.588 & 17,830 & (e) & 1.5 & 56,346 & (o) & 1.5 & & & & -0.84 \\
\hline 2599.198 & 15,084 & (e) & 3.5 & 53,546 & (o) & 4.5 & & & & -0.74 \\
\hline
\end{tabular}




\begin{tabular}{|c|c|}
\hline 2606.377 & 3051 \\
\hline 2607.248 & 14,359 \\
\hline 2613.616 & 17,389 \\
\hline 2614.295 & 15,254 \\
\hline 2620.930 & 15,084 \\
\hline 2622.747 & 3645 \\
\hline 2626.949 & 6344 \\
\hline 2635.783 & 17,711 \\
\hline 2638.718 & 0 \\
\hline 2641.410 & 8362 \\
\hline 2647.297 & 8362 \\
\hline 2649.132 & 14,359 \\
\hline 2651.159 & 17,369 \\
\hline 2657.488 & 15,084 \\
\hline 2657.847 & 4905 \\
\hline 2661.883 & 6344 \\
\hline 2665.977 & 12,921 \\
\hline 2671.240 & 13,486 \\
\hline 2676.607 & 17,711 \\
\hline 2677.555 & 6344 \\
\hline 2678.398 & 17,369 \\
\hline 2683.358 & 15,084 \\
\hline 2685.208 & 17,830 \\
\hline 2706.643 & 12,070 \\
\hline 2706.735 & 13,486 \\
\hline 2711.929 & 17,830 \\
\hline 2712.143 & 12,070 \\
\hline 2712.430 & 4905 \\
\hline 2718.494 & 14,359 \\
\hline 2731.164 & 17,369 \\
\hline 2732.671 & 17,389 \\
\hline 2738.765 & 4905 \\
\hline 2751.811 & 8362 \\
\hline 2756.913 & 17,711 \\
\hline 2770.439 & 12,921 \\
\hline 2772.332 & 14,359 \\
\hline 2773.356 & 6344 \\
\hline 2773.508 & 11,952 \\
\hline 2774.014 & 8362 \\
\hline 2786.300 & 15,254 \\
\hline 2789.495 & 17,389 \\
\hline 2789.710 & 17,711 \\
\hline 2789.828 & 12,070 \\
\hline 2808.003 & 4905 \\
\hline 2813.872 & 3051 \\
\hline 2814.480 & 13,486 \\
\hline 2814.760 & 17,711 \\
\hline 2816.059 & 17,830 \\
\hline 2820.231 & 3051 \\
\hline 2820.427 & 13,486 \\
\hline 2822.680 & 6344 \\
\hline 2828.135 & 17,369 \\
\hline 2829.325 & 17,369 \\
\hline 2849.212 & 12,070 \\
\hline
\end{tabular}

\begin{tabular}{|c|c|c|c|c|}
\hline (e) & 2.5 & 41,407 & (o) & 3.5 \\
\hline (e) & 1.5 & 52,703 & (o) & 2.5 \\
\hline (e) & 4.5 & 55,639 & (o) & 4.5 \\
\hline (e) & 0.5 & 53,494 & (o) & 1.5 \\
\hline (e) & 3.5 & 53,227 & (o) & 3.5 \\
\hline (e) & 1.5 & 41,761 & (o) & 2.5 \\
\hline (e) & 3.5 & 44,400 & (o) & 3.5 \\
\hline (e) & 3.5 & 55,639 & (o) & 4.5 \\
\hline (e) & 1.5 & 37,886 & (o) & 1.5 \\
\hline (e) & 4.5 & 46,209 & (o) & 5.5 \\
\hline (e) & 4.5 & 46,125 & (o) & 4.5 \\
\hline (e) & 1.5 & 52,096 & (o) & 0.5 \\
\hline (e) & 2.5 & 55,077 & (o) & 1.5 \\
\hline (e) & 3.5 & 52,703 & (o) & 2.5 \\
\hline (e) & 2.5 & 42,518 & (o) & 1.5 \\
\hline (e) & 3.5 & 43,901 & (o) & 2.5 \\
\hline (e) & 1.5 & 50,419 & (o) & 1.5 \\
\hline (e) & 2.5 & 50,910 & (o) & 2.5 \\
\hline (e) & 3.5 & 55,060 & (o) & 2.5 \\
\hline (e) & 3.5 & 43,681 & (o) & 2.5 \\
\hline (e) & 2.5 & 54,694 & (o) & 2.5 \\
\hline (e) & 3.5 & 52,340 & (o) & 3.5 \\
\hline (e) & 1.5 & 55,060 & (o) & 2.5 \\
\hline (e) & 2.5 & 49,006 & (o) & 2.5 \\
\hline (e) & 2.5 & 50,419 & (o) & 1.5 \\
\hline (e) & 1.5 & 54,694 & (o) & 2.5 \\
\hline (e) & 2.5 & 48,931 & (o) & 3.5 \\
\hline (e) & 2.5 & 41,761 & (o) & 2.5 \\
\hline (e) & 1.5 & 51,134 & (o) & 1.5 \\
\hline (e) & 2.5 & 53,972 & (o) & 3.5 \\
\hline (e) & 4.5 & 53,972 & (o) & 3.5 \\
\hline (e) & 2.5 & 41,407 & (o) & 3.5 \\
\hline (e) & 4.5 & 44,691 & (o) & 3.5 \\
\hline (e) & 3.5 & 53,972 & (o) & 3.5 \\
\hline (e) & 1.5 & 49,006 & (o) & 2.5 \\
\hline (e) & 1.5 & 50,419 & (o) & 1.5 \\
\hline (e) & 3.5 & 42,391 & (o) & 4.5 \\
\hline (e) & 0.5 & 47,996 & (o) & 0.5 \\
\hline (e) & 4.5 & 44,400 & (o) & 3.5 \\
\hline (e) & 0.5 & 51,134 & (o) & 1.5 \\
\hline (e) & 4.5 & 53,227 & (o) & 3.5 \\
\hline (e) & 3.5 & 53,546 & (o) & 4.5 \\
\hline (e) & 2.5 & 47,904 & (o) & 2.5 \\
\hline (e) & 2.5 & 40,507 & (o) & 2.5 \\
\hline (e) & 2.5 & 38,579 & (o) & 2.5 \\
\hline (e) & 2.5 & 49,006 & (o) & 2.5 \\
\hline (e) & 3.5 & 53,227 & (o) & 3.5 \\
\hline (e) & 1.5 & 53,331 & (o) & 0.5 \\
\hline (e) & 2.5 & 38,499 & (o) & 3.5 \\
\hline (e) & 2.5 & 48,931 & (o) & 3.5 \\
\hline (e) & 3.5 & 41,761 & (o) & 2.5 \\
\hline (e) & 2.5 & 52,717 & (o) & 1.5 \\
\hline (e) & 2.5 & 52,703 & (o) & 2.5 \\
\hline (e) & 2.5 & 47,158 & (o) & 3.5 \\
\hline
\end{tabular}

\begin{tabular}{|c|c|c|c|}
\hline & & & -0.35 \\
\hline & & & -0.32 \\
\hline & & & 0.35 \\
\hline & & & -0.86 \\
\hline & & & -0.87 \\
\hline & & & -0.23 \\
\hline & & & 0.16 \\
\hline & & & -0.30 \\
\hline & -0.17 & -0.40 & -0.25 \\
\hline & 0.57 & 0.61 & 0.65 \\
\hline & & & 0.30 \\
\hline & & & -0.78 \\
\hline & & & -0.08 \\
\hline & & & -0.62 \\
\hline-0.902 & & -0.96 & -0.85 \\
\hline & & & -0.66 \\
\hline & & & -0.36 \\
\hline & & & -0.68 \\
\hline & & & -0.82 \\
\hline-1.866 & & -1.05 & -0.77 \\
\hline & & & -0.79 \\
\hline 0.319 & & 0.36 & 0.35 \\
\hline & & & -0.35 \\
\hline & & & -0.69 \\
\hline & & & -0.43 \\
\hline & & & -0.52 \\
\hline & & & -0.92 \\
\hline & & & -0.65 \\
\hline & & & -0.73 \\
\hline & & & -0.76 \\
\hline & & & -0.83 \\
\hline & & & 0.00 \\
\hline & & & -0.38 \\
\hline & & & -0.13 \\
\hline & & & -0.99 \\
\hline & & & -0.53 \\
\hline & 0.30 & 0.29 & 0.38 \\
\hline & & & -0.56 \\
\hline & & & -0.45 \\
\hline & & & -0.83 \\
\hline & & & -0.05 \\
\hline & & & 0.26 \\
\hline-0.923 & & -0.74 & -0.71 \\
\hline & & & -0.64 \\
\hline & & & -0.84 \\
\hline & & & -0.19 \\
\hline & & & -0.21 \\
\hline & & & -0.87 \\
\hline-0.044 & -0.05 & -0.02 & 0.08 \\
\hline & & & -0.75 \\
\hline & & & -0.25 \\
\hline & & & -0.66 \\
\hline & & & $\begin{array}{l}-0.38 \\
-0.02\end{array}$ \\
\hline
\end{tabular}


Table 5 (continued)

\begin{tabular}{|c|c|c|c|c|c|c|c|c|c|c|}
\hline \multirow[t]{2}{*}{$\lambda(\AA)^{\mathrm{a}}$} & \multicolumn{3}{|c|}{ Lower level $^{\mathrm{b}}$} & \multicolumn{3}{|c|}{ Upper level $^{\mathrm{b}}$} & \multicolumn{2}{|c|}{$\begin{array}{c}\log g f \\
\text { Experiment }\end{array}$} & \multicolumn{2}{|c|}{$\begin{array}{l}\log g f^{c} \\
\text { This work }\end{array}$} \\
\hline & $E\left(\mathrm{~cm}^{-1}\right)$ & & $J$ & $E\left(\mathrm{~cm}^{-1}\right)$ & & $J$ & [3] & [4] & OSP & $\mathrm{HFR}+\mathrm{CPOL}$ \\
\hline 2850.152 & 12,921 & (e) & 1.5 & 47,996 & (o) & 0.5 & & & & -0.80 \\
\hline 2851.210 & 6344 & (e) & 3.5 & 41,407 & (o) & 3.5 & & & & -0.58 \\
\hline 2852.016 & 12,921 & (e) & 1.5 & 47,974 & (o) & 1.5 & & & & -0.48 \\
\hline 2857.655 & 12,921 & (e) & 1.5 & 47,904 & (o) & 2.5 & -0.850 & & -0.68 & -0.68 \\
\hline 2860.319 & 17,389 & (e) & 4.5 & 52,340 & (o) & 3.5 & -0.500 & & -0.24 & -0.33 \\
\hline 2861.016 & 0 & (e) & 1.5 & 34,942 & (o) & 2.5 & -0.693 & -0.69 & -0.63 & -0.55 \\
\hline 2861.702 & 3645 & (e) & 1.5 & 38,579 & (o) & 2.5 & & & & -0.13 \\
\hline 2876.340 & 15,084 & (e) & 3.5 & 49,840 & (o) & 4.5 & -0.062 & & -0.02 & -0.03 \\
\hline 2879.119 & 11,952 & (e) & 0.5 & 46,674 & (o) & 1.5 & -1.107 & & -0.95 & -0.98 \\
\hline 2885.471 & 14,359 & (e) & 1.5 & 49,006 & (o) & 2.5 & & & & -0.72 \\
\hline 2898.710 & 13,486 & (e) & 2.5 & 47,974 & (o) & 1.5 & & & & -0.33 \\
\hline 2904.535 & 13,486 & (e) & 2.5 & 47,904 & (o) & 2.5 & -0.947 & & -0.51 & -0.59 \\
\hline 2919.599 & 3645 & (e) & 1.5 & 37,886 & (o) & 1.5 & & -0.71 & -0.70 & -0.65 \\
\hline 2929.638 & 0 & (e) & 1.5 & 34,124 & (o) & 1.5 & -0.992 & -0.94 & -0.95 & -0.86 \\
\hline 2937.782 & 8362 & (e) & 4.5 & 42,391 & (o) & 4.5 & & -0.14 & -0.01 & -0.02 \\
\hline 2960.806 & 17,369 & (e) & 2.5 & 51,134 & (o) & 1.5 & & & & -0.53 \\
\hline 2961.798 & 12,921 & (e) & 1.5 & 46,674 & (o) & 1.5 & -0.712 & & -0.53 & -0.61 \\
\hline 2967.237 & 11,952 & (e) & 0.5 & 45,643 & (o) & 0.5 & -0.543 & & -0.51 & -0.51 \\
\hline 2968.803 & 4905 & (e) & 2.5 & 38,579 & (o) & 2.5 & & & & -0.39 \\
\hline 2975.882 & 4905 & (e) & 2.5 & 38,499 & (o) & 3.5 & -0.299 & -0.27 & -0.28 & -0.25 \\
\hline 2977.588 & 12,921 & (e) & 1.5 & 46,495 & (o) & 0.5 & -0.911 & & -0.80 & -0.85 \\
\hline 2990.807 & 23,146 & (e) & 4.5 & 56,572 & (o) & 5.5 & & & & -0.96 \\
\hline 3011.215 & 17,711 & (e) & 3.5 & 50,910 & (o) & 2.5 & & & & -0.55 \\
\hline 3012.975 & 0 & (e) & 1.5 & 33,180 & (o) & 2.5 & -0.681 & -0.60 & -0.62 & -0.54 \\
\hline 3025.286 & 8362 & (e) & 4.5 & 41,407 & (o) & 3.5 & & & & -0.72 \\
\hline 3031.162 & 4905 & (e) & 2.5 & 37,886 & (o) & 1.5 & & -0.48 & -0.47 & -0.40 \\
\hline 3046.024 & 15,084 & (e) & 3.5 & 47,904 & (o) & 2.5 & -0.996 & & -0.87 & -0.71 \\
\hline 3055.414 & 15,254 & (e) & 0.5 & 47,974 & (o) & 1.5 & & & & -0.77 \\
\hline 3064.689 & 12,070 & (e) & 2.5 & 44,691 & (o) & 3.5 & & & & -0.84 \\
\hline 3080.635 & 17,389 & (e) & 4.5 & 49,840 & (o) & 4.5 & 0.083 & & 0.11 & 0.13 \\
\hline 3092.252 & 12,070 & (e) & 2.5 & 44,400 & (o) & 3.5 & & & & -0.79 \\
\hline 3101.386 & 6344 & (e) & 3.5 & 38,579 & (o) & 2.5 & & & & -0.62 \\
\hline 3109.113 & 6344 & (e) & 3.5 & 38,499 & (o) & 3.5 & -0.288 & -0.26 & -0.29 & -0.24 \\
\hline 3110.877 & 14,359 & (e) & 1.5 & 46,495 & (o) & 0.5 & -0.513 & & -0.68 & -0.52 \\
\hline 3134.725 & 3051 & (e) & 2.5 & 34,942 & (o) & 2.5 & -0.458 & -0.42 & -0.42 & -0.40 \\
\hline 3140.770 & 12,070 & (e) & 2.5 & 43,901 & (o) & 2.5 & & & & -0.39 \\
\hline 3176.855 & 4905 & (e) & 2.5 & 36,373 & (o) & 1.5 & & -0.80 & -1.06 & -0.93 \\
\hline 3193.531 & 3051 & (e) & 2.5 & 34,355 & (o) & 2.5 & -0.939 & -0.89 & -1.09 & -0.74 \\
\hline 3194.198 & 3645 & (e) & 1.5 & 34,942 & (o) & 2.5 & -0.509 & -0.49 & -0.48 & -0.48 \\
\hline 3199.989 & 15,254 & (e) & 0.5 & 46,495 & (o) & 0.5 & -0.629 & & -0.67 & -0.56 \\
\hline 3202.146 & 17,711 & (e) & 3.5 & 48,931 & (o) & 3.5 & & & & -0.96 \\
\hline 3218.167 & 21,638 & (e) & 3.5 & 52,703 & (o) & 2.5 & & & & -0.79 \\
\hline 3220.654 & 15,084 & (e) & 3.5 & 46,125 & (o) & 4.5 & & & & -0.37 \\
\hline 3253.702 & 3051 & (e) & 2.5 & 33,776 & (o) & 3.5 & -0.828 & -0.81 & -0.83 & -0.77 \\
\hline 3294.663 & 28,105 & (e) & 4.5 & 58,448 & (o) & 3.5 & & & & -0.93 \\
\hline 3323.324 & 23,146 & (e) & 4.5 & 53,227 & (o) & 3.5 & & & & -0.35 \\
\hline 3333.480 & 28,458 & (e) & 3.5 & 58,448 & (o) & 3.5 & & & & -0.93 \\
\hline
\end{tabular}




$\begin{array}{lr}3352.051 & 8362 \\ 3358.289 & 17,389 \\ 3370.662 & 28,458 \\ 3376.671 & 15,084 \\ 3389.829 & 3645 \\ 3394.974 & 17,711 \\ 3399.793 & 0 \\ 3407.757 & 12,070 \\ 3469.265 & 15,084 \\ 3478.980 & 17,389 \\ 3487.573 & 17,830 \\ 3495.745 & 6344 \\ 3505.219 & 8362 \\ 3511.862 & 28,105 \\ 3518.740 & 14,359 \\ 3535.649 & 4905 \\ 3561.659 & 0 \\ 3569.034 & 6344 \\ 3599.110 & 18,898 \\ 3599.305 & 27,285 \\ 3600.052 & 20,135 \\ 3644.352 & 6344 \\ 3659.032 & 17,369 \\ 3661.045 & 15,084 \\ 3661.738 & 17,389 \\ 3665.349 & 11,952 \\ 3699.731 & 13,486 \\ 3718.769 & 30,595 \\ 3719.276 & 4905 \\ 3737.869 & 18,898 \\ 3744.969 & 23,146 \\ 3745.763 & 17,711 \\ 3758.021 & 28,458 \\ 3762.507 & 31,878 \\ 3766.909 & 20,135 \\ 3770.621 & 28,547 \\ 3797.938 & 15,084 \\ 3806.062 & 21,638 \\ 3810.566 & 28,458 \\ 3817.191 & 17,711 \\ 3823.522 & 28,547 \\ 3834.706 & 17,830 \\ 3864.740 & 28,105 \\ 3877.098 & 23,146 \\ 3882.227 & 30,595 \\ 3900.627 & 30,942 \\ 3917.447 & 21,638 \\ 3923.902 & 12,921 \\ 3935.632 & 17,369 \\ 3945.331 & 32,778 \\ 3979.379 & 28,105 \\ 3984.841 & 28,458 \\ 4007.357 & 28,947 \\ 4047.959 & \end{array}$

\begin{tabular}{|c|c|c|c|c|}
\hline (e) & 4.5 & 38,186 & (o) & 4.5 \\
\hline (e) & 4.5 & 47,158 & (o) & 3.5 \\
\hline (e) & 3.5 & 58,117 & (o) & 2.5 \\
\hline (e) & 3.5 & 44,691 & (o) & 3.5 \\
\hline (e) & 1.5 & 33,136 & (o) & 0.5 \\
\hline (e) & 3.5 & 47,158 & (o) & 3.5 \\
\hline (e) & 1.5 & 29,405 & (o) & 2.5 \\
\hline (e) & 2.5 & 41,407 & (o) & 3.5 \\
\hline (e) & 3.5 & 43,901 & (o) & 2.5 \\
\hline (e) & 4.5 & 46,125 & (o) & 4.5 \\
\hline (e) & 1.5 & 46,495 & (o) & 0.5 \\
\hline (e) & 3.5 & 34,942 & (o) & 2.5 \\
\hline (e) & 4.5 & 36,882 & (o) & 3.5 \\
\hline (e) & 4.5 & 56,572 & (o) & 5.5 \\
\hline (e) & 1.5 & 42,771 & (o) & 1.5 \\
\hline (e) & 2.5 & 33,180 & (o) & 2.5 \\
\hline (e) & 1.5 & 28,069 & (o) & 1.5 \\
\hline (e) & 3.5 & 34,355 & (o) & 2.5 \\
\hline (e) & 1.5 & 46,674 & (o) & 1.5 \\
\hline (e) & 1.5 & 55,060 & (o) & 2.5 \\
\hline (e) & 2.5 & 47,904 & (o) & 2.5 \\
\hline (e) & 3.5 & 33,776 & (o) & 3.5 \\
\hline (e) & 2.5 & 44,691 & (o) & 3.5 \\
\hline (e) & 3.5 & 42,391 & (o) & 4.5 \\
\hline (e) & 4.5 & 44,691 & (o) & 3.5 \\
\hline (e) & 0.5 & 39,226 & (o) & 1.5 \\
\hline (e) & 2.5 & 40,507 & (o) & 2.5 \\
\hline (e) & 1.5 & 57,478 & (o) & 0.5 \\
\hline (e) & 2.5 & 31,784 & (o) & 1.5 \\
\hline (e) & 1.5 & 45,643 & (o) & 0.5 \\
\hline (e) & 4.5 & 49,840 & (o) & 4.5 \\
\hline (e) & 3.5 & 44,400 & (o) & 3.5 \\
\hline (e) & 3.5 & 55,060 & (o) & 2.5 \\
\hline (e) & 4.5 & 58,448 & (o) & 3.5 \\
\hline (e) & 2.5 & 46,674 & (o) & 1.5 \\
\hline (e) & 2.5 & 55,060 & (o) & 2.5 \\
\hline (e) & 3.5 & 41,407 & (o) & 3.5 \\
\hline (e) & 3.5 & 47,904 & (o) & 2.5 \\
\hline (e) & 3.5 & 54,694 & (o) & 2.5 \\
\hline (e) & 3.5 & 43,901 & (o) & 2.5 \\
\hline (e) & 2.5 & 54,694 & (o) & 2.5 \\
\hline (e) & 1.5 & 43,901 & (o) & 2.5 \\
\hline (e) & 4.5 & 53,972 & (o) & 3.5 \\
\hline (e) & 4.5 & 48,931 & (o) & 3.5 \\
\hline (e) & 1.5 & 56,346 & (o) & 1.5 \\
\hline (e) & 5.5 & 56,572 & (o) & 5.5 \\
\hline (e) & 3.5 & 47,158 & (o) & 3.5 \\
\hline (e) & 1.5 & 38,399 & (o) & 0.5 \\
\hline (e) & 2.5 & 42,771 & (o) & 1.5 \\
\hline (e) & 2.5 & 58,117 & (o) & 2.5 \\
\hline (e) & 4.5 & 53,227 & (o) & 3.5 \\
\hline (e) & 3.5 & 53,546 & (o) & 4.5 \\
\hline (e) & 2.5 & 53,494 & (o) & 1.5 \\
\hline (e) & 5.5 & 55,639 & (o) & 4.5 \\
\hline
\end{tabular}

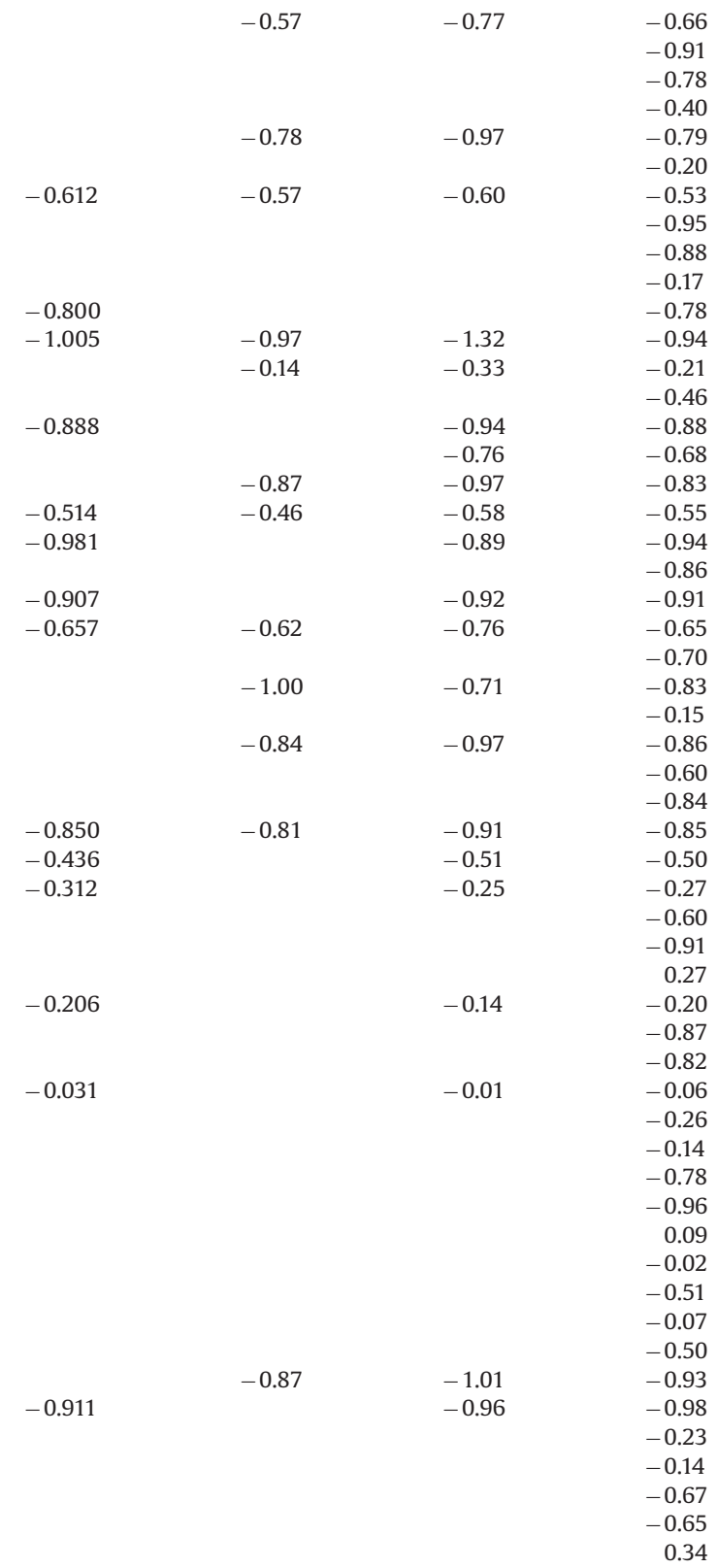




\begin{tabular}{|c|c|c|c|c|c|c|c|c|c|c|}
\hline \multirow[t]{2}{*}{$\lambda(\AA)^{\mathrm{a}}$} & \multicolumn{3}{|c|}{ Lower level $^{\mathrm{b}}$} & \multicolumn{3}{|c|}{ Upper level $^{\mathrm{b}}$} & \multicolumn{2}{|c|}{$\begin{array}{c}\log g f \\
\text { Experiment }\end{array}$} & \multicolumn{2}{|c|}{$\begin{array}{l}\log g f^{c} \\
\text { This work }\end{array}$} \\
\hline & $E\left(\mathrm{~cm}^{-1}\right)$ & & $J$ & $E\left(\mathrm{~cm}^{-1}\right)$ & & $J$ & [3] & {$[4]$} & OSP & $\mathrm{HFR}+\mathrm{CPOL}$ \\
\hline 4048.444 & 31,878 & (e) & 4.5 & 56,572 & (o) & 5.5 & & & & -0.81 \\
\hline 4123.495 & 28,458 & (e) & 3.5 & 52,703 & (o) & 2.5 & & & & -0.60 \\
\hline 4125.083 & 28,105 & (e) & 4.5 & 52,340 & (o) & 3.5 & -0.953 & & & -0.95 \\
\hline 4127.789 & 14,359 & (e) & 1.5 & 38,579 & (o) & 2.5 & & & & -0.97 \\
\hline 4162.405 & 17,389 & (e) & 4.5 & 41,407 & (o) & 3.5 & & & & -0.91 \\
\hline 4177.520 & 17,830 & (e) & 1.5 & 41,761 & (o) & 2.5 & & & & -0.74 \\
\hline 4232.426 & 18,898 & (e) & 1.5 & 42,518 & (o) & 1.5 & -0.631 & & -0.42 & -0.50 \\
\hline 4241.923 & 32,778 & (e) & 2.5 & 56,346 & (o) & 1.5 & & & & -0.81 \\
\hline 4245.845 & 20,135 & (e) & 2.5 & 43,681 & (o) & 2.5 & -1.154 & & -0.40 & -0.41 \\
\hline 4321.359 & 27,285 & (e) & 1.5 & 50,419 & (o) & 1.5 & & & & -0.51 \\
\hline 4334.640 & 23,146 & (e) & 4.5 & 46,209 & (o) & 5.5 & & -0.59 & -0.66 & -0.74 \\
\hline 4350.516 & 23,146 & (e) & 4.5 & 46,125 & (o) & 4.5 & & & & -0.16 \\
\hline 4392.055 & 21,638 & (e) & 3.5 & 44,400 & (o) & 3.5 & & & & -0.23 \\
\hline 4417.358 & 15,254 & (e) & 0.5 & 37,886 & (o) & 1.5 & & -1.00 & -0.95 & -0.88 \\
\hline 4422.715 & 30,942 & (e) & 5.5 & 53,546 & (o) & 4.5 & & & & 0.03 \\
\hline 4426.173 & 28,547 & (e) & 2.5 & 51,134 & (o) & 1.5 & & & & -0.75 \\
\hline 4483.280 & 32,778 & (e) & 2.5 & 55,077 & (o) & 1.5 & & & & -0.88 \\
\hline 4486.630 & 32,778 & (e) & 2.5 & 55,060 & (o) & 2.5 & & & & -0.68 \\
\hline 4524.699 & 31,878 & (e) & 4.5 & 53,972 & (o) & 3.5 & & & & -0.66 \\
\hline 4535.361 & 21,638 & (e) & 3.5 & 43,681 & (o) & 2.5 & -1.133 & & -0.83 & -0.86 \\
\hline 4570.683 & 28,547 & (e) & 2.5 & 50,419 & (o) & 1.5 & & & & -0.95 \\
\hline 4599.450 & 28,105 & (e) & 4.5 & 49,840 & (o) & 4.5 & -0.504 & & -0.38 & -0.46 \\
\hline 4613.717 & 31,878 & (e) & 4.5 & 53,546 & (o) & 4.5 & & & & -0.16 \\
\hline 4622.705 & 20,135 & (e) & 2.5 & 41,761 & (o) & 2.5 & & & & -0.77 \\
\hline 4675.454 & 28,458 & (e) & 3.5 & 49,840 & (o) & 4.5 & -1.048 & & -0.74 & -0.92 \\
\hline 4817.206 & 21,638 & (e) & 3.5 & 42,391 & (o) & 4.5 & & & -0.72 & -0.82 \\
\hline 4834.767 & 37,440 & (e) & 3.5 & 58,117 & (o) & 2.5 & & & & -0.86 \\
\hline 4865.418 & 28,458 & (e) & 3.5 & 49,006 & (o) & 2.5 & & & & -0.83 \\
\hline 4904.511 & 28,547 & (e) & 2.5 & 48,931 & (o) & 3.5 & & & & -0.59 \\
\hline 5110.588 & 32,778 & (e) & 2.5 & 52,340 & (o) & 3.5 & -0.908 & & -0.73 & -0.96 \\
\hline 5247.128 & 28,105 & (e) & 4.5 & 47,158 & (o) & 3.5 & & & & -0.65 \\
\hline 5276.328 & 37,398 & (e) & 2.5 & 56,346 & (o) & 1.5 & & & & -0.87 \\
\hline 5346.275 & 28,458 & (e) & 3.5 & 47,158 & (o) & 3.5 & & & & -0.70 \\
\hline 5493.239 & 37,440 & (e) & 3.5 & 55,639 & (o) & 4.5 & & & & -0.87 \\
\hline 5673.572 & 37,440 & (e) & 3.5 & 55,060 & (o) & 2.5 & & & & -0.58 \\
\hline 6027.552 & 28,105 & (e) & 4.5 & 44,691 & (o) & 3.5 & & & & -0.89 \\
\hline 6473.888 & 28,458 & (e) & 3.5 & 43,901 & (o) & 2.5 & & & & -0.99 \\
\hline 6709.435 & 37,440 & (e) & 3.5 & 52,340 & (o) & 3.5 & & & -0.98 & -0.76 \\
\hline
\end{tabular}

a Wavelengths deduced from experimental energy levels. They are given in air for $\lambda$ longer than $2000 \AA$ and in vacuum for $\lambda$ shorter than $2000 \AA$.

b Experimental levels from $[8,15]$. (e) and (o) stand for even and odd parities, respectively.

c OSP: oscillator strength parametrization method; HFR+CPOL: pseudo-relativistic Hartree-Fock method with core-polarization corrections (see text). 
useful guide for experimenters to perform further spectroscopic measurements in this ion.

\section{Acknowledgments}

PP and PQ are respectively Research Director and Research Associate of the Belgian Fund for Scientific Research F.R.S.-FNRS. Financial support from this organization is gratefully acknowledged.

\section{Appendix A. Supplementary material}

Supplementary data associated with this article can be found in the online version at http://dx.doi.org/10.1016/j. jqsrt.2015.04.013.

\section{References}

[1] Bouazza S, Fienhold M, Guthöhrlein GH, Behrens HO, Dembczynski J. Doppler limited laser spectroscopy on hafnium lines. Part I: Hyperfine structure of even-parity levels. Eur Phys J D 1999;6:303.

[2] Bouazza S, Behrens HO, Fienhold M, Dembczynski J, Guthöhrlein GH. Doppler limited laser spectroscopy on hafnium lines. Part II: Hyperfine structure of odd-parity levels. Eur Phys J D 1999;6:311.

[3] Lundqvist M, Nilsson H, Wahlgren GM, Lundberg H, Xu HL, Jang Z $\mathrm{K}$, et al. Improved oscillator strengths and wavelengths in $\mathrm{Hf} \mathrm{II}$, with applications to stellar elemental abundances. Astron Astrophys 2006;450:407. http://dx.doi.org/10.1051/0004-6361:20054474.

[4] Lawler JE, Den Hartog EA, Labby ZE, Sneden C, Cowan JJ, Ivans II. Improved laboratory transition probabilities for Hf II and hafnium abundances in the sun and 10-metal-poor stars. Astrophys J Suppl Ser 2007;169 [1200049/214/2/18].

[5] Kurucz RL. 〈http://kurucz.harvard.edu/atoms〉 [online].

[6] Ruczkowski J, Elantkowska M, Dembczynski J. An alternative method for determination of oscillator strengths: the example of Sc II. J Quant Spectr Radiat Transfer 2014;145:20. http://dx.doi.org/10.1016/ j.jqsit.2014.04.0148.

[7] Wyart JF. Étude systématique des configurations électroniques $(5 d+6 s)^{N}$ dans les éléments une fois ionisés du groupe du platine. Opt Pura Y Apl 1977;10:177.

[8] Wyart JF, Blaise J. Configuration mixing in singly-ionized platinum group elements. I. Low odd configurations of Hf II, Ta II and W II. Phys Scr 1990;42:209.
[9] Bouazza S. Investigations of hyperfine structure and isotope shift predictions in Hf II. Int J Quant Chem 2012;112:470. http://dx.doi.org/ 10.1002/qua.22974.

[10] Cowan RD. The theory of atomic structure and spectra. Berkeley: Berkeley University of California Press; 1981.

[11] Quinet P, Palmeri P, Biémont E, McCurdy MM, Rieger G, Pinnington $\mathrm{EH}$, et al. Experimental and theoretical lifetimes, branching fractions and oscillator strengths in LuII. Mon Not R Astr Soc 1999;307:934.

[12] Quinet P, Palmeri P, Biémont E, Li ZS, Zhang ZG, Svanberg S. Radiative lifetime measurements and transition probability calculations in lanthanide ions. J Alloys Compds 2002;344:255.

[13] Fivet V, Biémont E, Engström L, Lundberg H, Nilsson H, Palmeri P, et al. Radiative lifetime measurements and calculations in doubly ionized tantalum (Ta III). J Phys B: At Mol Opt Phys 2008;41:015702.

[14] Fraga S, Karwowski J, Saxena KMS. Handbook of atomic data. Amsterdam: Elsevier Scientific Publishing Company; 1976.

[15] 〈http://physics.nist.gov/asd〉.

[16] Quinet P, Fivet V, Palmeri P, Biémont E, Engström L, Lundberg H, et al. Branching fractions and A-values in singly ionized tanatlum (Ta II). Astron Astrophys 2009;493:711.

[17] Nilsson H, Engström L, Lundberg H, Palmeri P, Fivet V, Quinet P, et al. Lifetime measurements and transition probability calculations in singly ionized tungsten (W II). Eur Phys J D 2008;49:13.

[18] Palmeri P, Quinet P, Biémont E, Xu HL, Svanberg S. Transition probabilities and lifetimes in singly ionized rhenium. Mon Not $\mathrm{R}$ Astr Soc 2005;362:1348.

[19] Quinet P, Palmeri P, Biémont E, Jorissen A, Van Eck S, Svanberg S, et al. Transition probabilities and lifetimes in neutral and singly ionized osmium and the solar osmium abundance. Astron Astrophys 2006;448:1207. http://dx.doi.org/10.1051/0004-6361:20053852.

[20] Xu HL, Svanberg S, Quinet P, Palmeri P, Biémont E. Improved atomic data for iridium atom (Ir I) and ion (Ir II) and the solar content of iridium. J Quant Spectrosc Radiat Transfer 2007;104:52.

[21] Quinet P, Palmeri P, Fivet V, Biémont E, Nilsson H, Engström L, et al. Laser-induced-fluorescence lifetime measurements and relativistic Hartree-Fock oscillator strength calculations in singly ionized platinum. Phys Rev A 2008;77:022501.

[22] Fivet V, Quinet P, Biémont E, Xu HL. Transition probabilities and lifetimes in gold (Au I and Au II). J Phys B: At Mol Opt Phys 2006;39: 3587.

[23] Ruczkowski J, Bouazza S, Elantkowska M, Dembczynski J. Semimpirical analysis of oscillator strengths for Nb II. J Quant Spectrosc Radiat Transfer 2015;155:20. http://dx.doi.org/10.1016/j.jqsrt.2014. 12.014 .

[24] Bouazza S, Ruczkowski J, Elantkowska M, Dembczynski J. Hyperfine structure, lifetime and oscillator strength studies of V II. J Quant Spectrosc Radiat Transfer 2015, submitted. 